\title{
Small bowel MR enterography: problem solving in Crohn's disease
}

\author{
Nyree Griffin • Lee Alexander Grant • \\ Simon Anderson • Peter Irving • Jeremy Sanderson
}

Received: 10 December 2011 /Revised: 5 February 2012 / Accepted: 20 February 2012 /Published online: 17 March 2012

(C) European Society of Radiology 2012

\begin{abstract}
Magnetic resonance enterography (MRE) is fast becoming the first-line radiological investigation to evaluate the small bowel in patients with Crohn's disease. It can demonstrate both mural and extramural complications. The lack of ionizing radiation, together with high-contrast resolution, multiplanar capability and cine-imaging make it an attractive imaging modality in such patients who need prolonged follow-up. A key question in the management of such patients is the assessment of disease activity. Clinical indices, endoscopic and histological findings have traditionally been used as surrogate markers but all have limitations. MRE can help address this question. The purpose of this pictorial review is to (1) detail the MRE protocol used at our institution; (2) describe the rationale for the MR sequences used and their limitations; (3) compare MRE with other small bowel imaging techniques; (4) discuss how MRE can help distinguish between inflammatory, stricturing and penetrating disease, and thus facilitate management of this difficult condition.
\end{abstract}

\section{N. Griffin $(\triangle)$}

Department of Radiology, Guy's \& St Thomas' Hospital,

Westminster Bridge Road,

London SE1 7EH, UK

e-mail: nyreegriffin100@gmail.com

\section{A. Grant}

Department of Radiology, Royal Free Hospital,

Pond Street,

London NW3 2QG, UK

S. Anderson $\cdot$ P. Irving $\cdot$ J. Sanderson

Department of Gastroenterology, Guy's \& St Thomas' Hospital,

Westminster Bridge Road,

London SE1 7EH, UK
Main Messages

- MR enterography (MRE) is the preferred imaging investigation to assess Crohn's disease. T2-weighted, post-contrast and diffusion-weighted imaging (DWI) can be used.

- MRE offers no radiation exposure, high-contrast resolution, multiplanar ability and cine imaging.

- MRE can help define disease activity, a key question in the management of Crohn's disease.

- MRE can help distinguish between inflammatory, stricturing and penetrating disease.

- MRE can demonstrate both mural and extramural complications.

Keywords Crohn's disease · MRI · Small bowel . Inflammation

\section{Introduction}

Magnetic resonance enterography (MRE) is a radiological technique that has evolved in the last decade. It involves the use of magnetic resonance imaging (MRI) to assess the small bowel, following distension with an oral contrast agent. The advantages of this technique are that it involves no ionising radiation, is capable of multi-planar imaging, affords high-contrast resolution (with more detailed evaluation of bowel wall changes) and allows for cine-imaging. Its main indication at present is to evaluate small bowel involvement in patients with Crohn's disease (CD).

$\mathrm{CD}$ is a chronic inflammatory bowel condition with onset usually in young adulthood. Twenty to thirty percent of patients are younger than 20 years old. The prevalence in the UK is estimated to be about 150 cases per 100,000 population [1]. It can involve any part of the gastrointestinal tract. The small bowel, in particular the terminal ileum, is most commonly involved but perianal disease can occur in 
Table 1 Parameters for MRI protocol

\begin{tabular}{|c|c|c|c|c|c|c|c|c|}
\hline \multirow[t]{2}{*}{ Parameter } & \multicolumn{2}{|c|}{ (1) True FISP } & \multirow{2}{*}{$\begin{array}{l}\text { (2) Cine True FISP } \\
\text { Coronal }\end{array}$} & \multirow{2}{*}{$\begin{array}{l}\text { (3) Diffusion } \\
\text { Axial }\end{array}$} & \multicolumn{2}{|c|}{$\begin{array}{l}\text { (4) T1 volumetric fat-saturated } \\
\text { sequence }{ }^{a, b}\end{array}$} & \multicolumn{2}{|c|}{ (5) HASTE } \\
\hline & Axial & Coronal & & & Axial & Coronal & Axial & Coronal $^{\mathrm{c}}$ \\
\hline TR/TE (ms) & $4.09 / 1.77$ & $4.19 / 2.1$ & $74 / 1.48$ & $5,600 / 82$ & $5.43 / 2.41$ & $5.43 / 2.36$ & $900 / 83$ & $1,000 / 217$ \\
\hline Flip angle (degrees) & 53 & 70 & 63 & - & 10 & 10 & 150 & 139 \\
\hline Field of view (mm) & 400 & 450 & 450 & 350 & 400 & 450 & 400 & 400 \\
\hline Parallel imaging factor & 2 & 0 & 2 & 2 & 2 & 2 & 2 & 2 \\
\hline Section thickness (mm) & 4 & 4 & 8 & 5 & 3 & 3 & 6 & 5 \\
\hline Intersection gap (mm) & 0 & 3 & 1.6 & 0 & 0 & 0 & 0 & 0 \\
\hline Number of sections per stack & 50 & 20 & 18 & $40-60$ & 64 & 64 & 20 & 20 \\
\hline Breath-hold time per stack & 18 & 14 & Free breathing & Free breathing & 18 & 14 & 18 & 22 \\
\hline Number of stacks & 2 or 3 & 1 & 1 & 2 & 2 or 3 & 1 & 2 or 3 & 1 or 2 \\
\hline Bandwidth (Hz) & 454 & 488 & 965 & 1302 & 300 & 300 & 416 & 130 \\
\hline
\end{tabular}

True FISP true fast imaging with steady state precession, HASTE half-Fourier acquired single shot turbo spin echo, TR repetition time, TE echo time ${ }^{a}$ The axial and coronal sequences are performed before and following intravenous administration of gadolinium

${ }^{b}$ These sequences are performed following intravenous spasmolytic (e.g. buscopan) unless contraindicated

${ }^{\mathrm{c}}$ The coronal HASTE sequence can be performed with fat saturation to help distinguish between submucosal fat and oedema

up to $40 \%$. It is characterised histologically by the presence of non-caseating granulomas and trans-mural inflammation within the bowel, leading to erosions, ulceration and inflammatory stenosis. Enteric sinuses, fistulae, mesenteric phlegmons and abscess collections may complicate penetrating disease. With time, inflammation may become chronic and eventually lead to the development of fibrostenotic strictures.

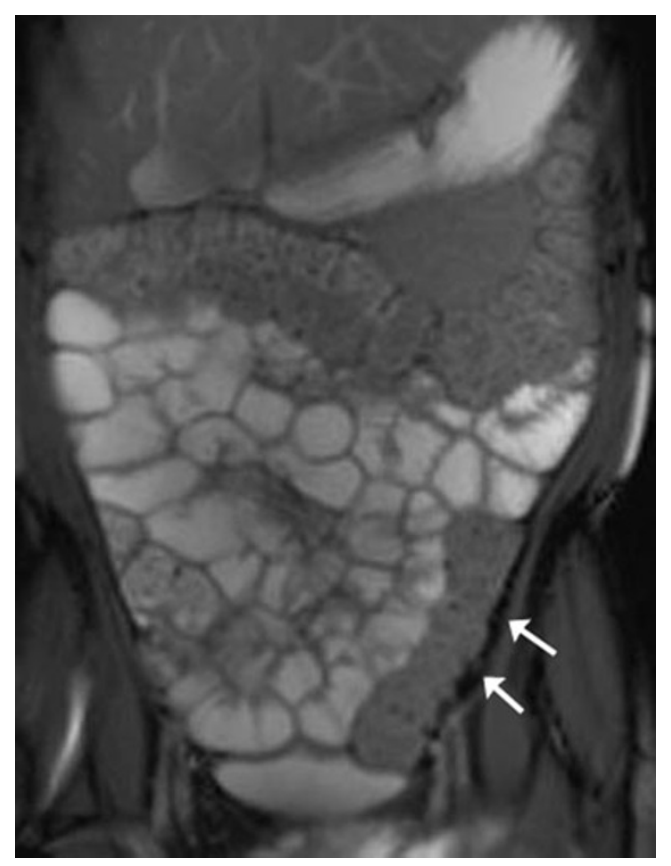

Fig. 1 Coronal True FISP image: normal bowel. The 'black boundary' artefact may be confused with bowel wall thickening (arrows)
The European Crohn's and Colitis Organisation (ECCO) has recently published a revised consensus on the diagnosis and management of $\mathrm{CD}$ [2]. Based on a systematic review of the literature, it recommends either computed tomography (CT) or MRE/MR enteroclysis as the current standards for assessing the small intestine due to their high diagnostic accuracy. It states that radiation exposure should be considered when selecting techniques. MRE can be used to help

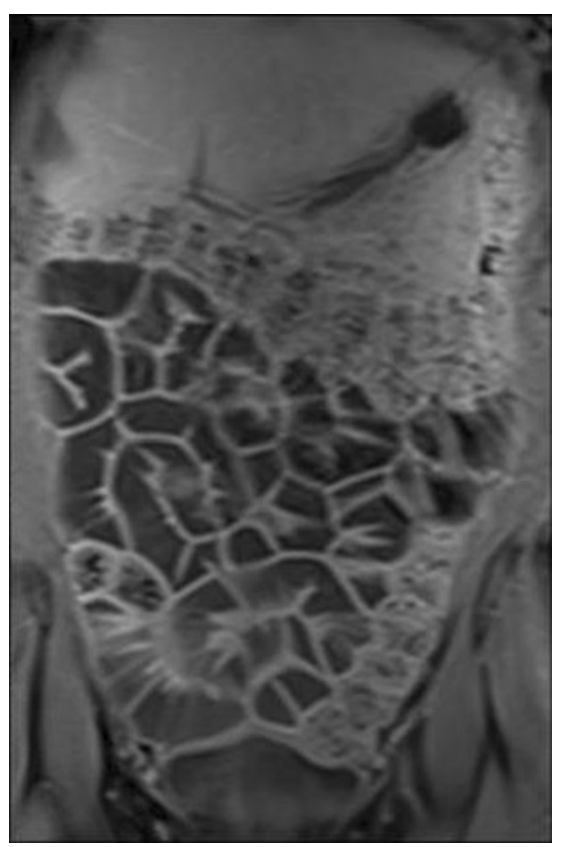

Fig. 2 Coronal T1 fat-saturated post-contrast image: normal bowel wall shows mild homogeneous enhancement 


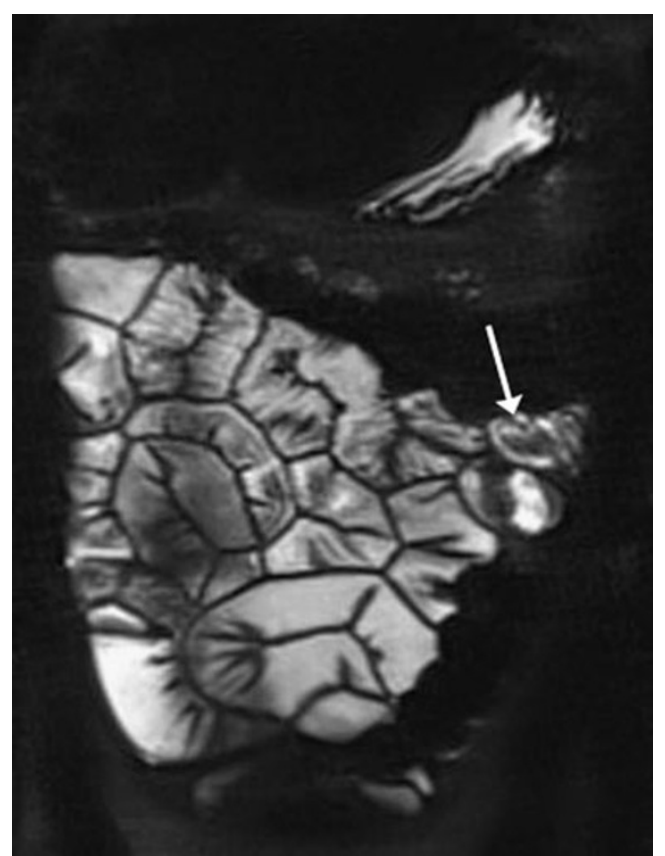

Fig. 3 Coronal fat-saturated HASTE image: normal bowel. Intraluminal flow voids (arrow) are seen, as this sequence is sensitive to fluid motion

categorise the relative components of inflammatory, penetrating or stricturing disease in each individual patient. This advantage in combination with no radiation exposure makes MRE an ideal investigation for repeat bowel imaging in patients with $\mathrm{CD}$.
A recent study looking into the diagnostic and therapeutic impact of MRE in CD has shown that MRE significantly increased the mean percentage confidence of clinicians for the presence or absence of small bowel disease and changed therapeutic strategy in $61 \%$ of patients [3].

The purpose of this pictorial review is to (1) detail the MRE protocol used at our institution; (2) describe the rationale for the MR sequences used and their limitations; (3) compare MRE with other small bowel imaging techniques; (4) discuss how MRE can assist the clinician in problem solving in $\mathrm{CD}$.

\section{MRE protocol}

The patient is fasted for 4-6 h prior to the study. In adults, oral contrast consists of at least 1-1.5 1 of a $2.5 \%$ mannitol solution mixed with $0.2 \%$ carob bean gum. This solution acts as a hyperosmolar agent to draw fluid into the bowel. In paediatric patients, the volume of oral contrast is adjusted (between 300-1,000 ml) according to the weight of the patient. Mannitol is a biphasic agent that appears as low signal intensity on T1-weighted images and high signal intensity on T2-weighted images. The patient drinks the oral contrast agent at regular intervals over a period of approximately $40 \mathrm{~min}$ prior to the study. The patient is imaged on a 1.5-T MRI scanner (Siemens, Erlangen, Germany), using a phased array surface coil, either in the supine or prone

Table 2 Comparison of imaging modalities in the evaluation of Crohn's disease

\begin{tabular}{|c|c|c|}
\hline Imaging Modality & Advantages & Disadvantages \\
\hline Barium follow-through & $\begin{array}{l}\text { Good depiction of early bowel disease } \\
\text { compared to CTE/MRE }\end{array}$ & $\begin{array}{l}\text { Radiation burden; time consuming, operator and } \\
\text { patient dependent leading to limited sensitivity; } \\
\text { difficulty in assessing extramural complications }\end{array}$ \\
\hline Ultrasound & $\begin{array}{l}\text { No radiation; may show terminal ileal } \\
\text { disease well }\end{array}$ & $\begin{array}{l}\text { Operator and patient dependent; comprehensive } \\
\text { examination is not possible; time consuming }\end{array}$ \\
\hline CTE & $\begin{array}{l}\text { Fast }(<5 \text { mins); greater spatial resolution than } \\
\text { MRE; multiplanar reformats are possible; } \\
\text { mural and extramural complications are seen }\end{array}$ & $\begin{array}{l}\text { Radiation burden; early disease is not well seen; } \\
\text { cine imaging is not possible }\end{array}$ \\
\hline MRE & $\begin{array}{l}\text { No radiation; high soft tissue contrast; multiplanar } \\
\text { ability; shows mural and extramural complications; } \\
\text { defines activity of disease; cine imaging is possible; } \\
\text { can combine with perianal imaging }\end{array}$ & $\begin{array}{l}\text { Longer scan time than CTE ( } 20 \text { mins); early disease } \\
\text { is not well seen; suboptimal distention of proximal } \\
\text { small bowel }\end{array}$ \\
\hline $\begin{array}{l}\text { Enteroclysis (barium/CT/ } \\
\text { MRI) }\end{array}$ & $\begin{array}{l}\text { Very good distension; can identify early ulceration, } \\
\text { wall thickening, fistulae, sinus tracts }\end{array}$ & Radiation burden; invasive; time consuming \\
\hline $\begin{array}{l}\text { Nuclear medicine } \\
\text { techniques }^{\mathrm{a}}\end{array}$ & Similar diagnostic accuracy to CTE and MRE & $\begin{array}{l}\text { Radiation burden; time consuming; poor localisation } \\
\text { (unless PET-CT) }\end{array}$ \\
\hline Balloon enteroscopy & Evaluate small bowel mucosa; biopsy is possible & $\begin{array}{l}\text { Requires sedation/anesthesia; extramural complications } \\
\text { not assessed; risk of pancreatitis, bleeding, small bowel } \\
\text { perforation }\end{array}$ \\
\hline Capsule endoscopy & Evaluate small bowel mucosa & $\begin{array}{l}\text { Cannot use in stricturing disease; battery exhaustion; poo } \\
\text { localisation; extramural complications are not assessed }\end{array}$ \\
\hline
\end{tabular}

CTE CT enterography, MRE MR enterography

${ }^{a}$ White cell scintigraphy, positron emission tomography 
position (if no stoma is present). Coronal sequences are quicker to perform in the prone position, as there is a thinner volume of tissue to image. Although prone imaging has been shown to result in better small bowel distension, both positions are equivalent with respect to lesion detection and characterisation of changes in the bowel wall in CD [4].
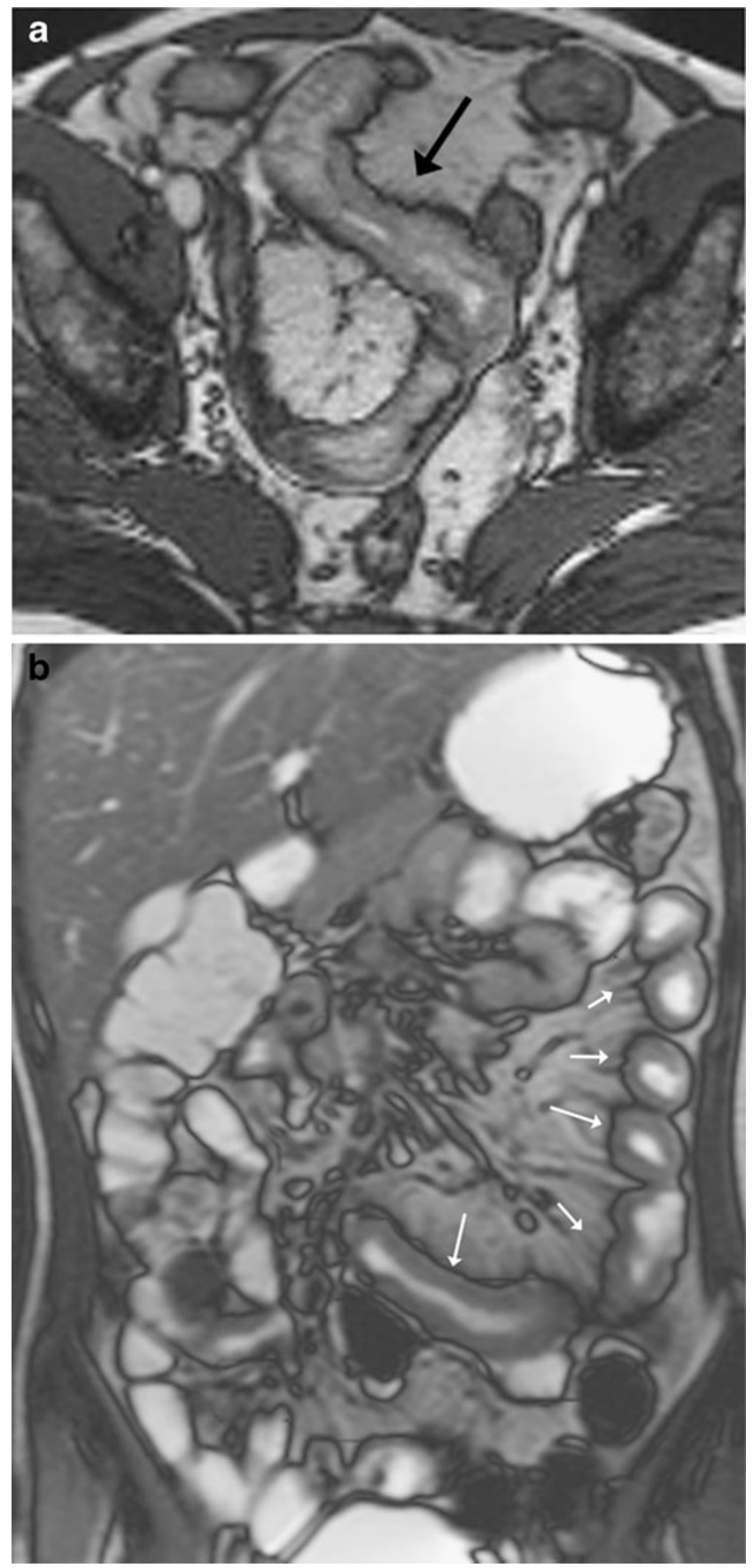

Fig. 4 Example of mural thickening in active Crohn's disease: a axial True FISP image shows mural thickening in the distal ileum (arrow); $\mathbf{b}$ coronal True FISP in a different patient ( 15 years old) shows extensive jejunal small bowel wall thickening (arrows); this distribution of disease is less common than distal/terminal ileum. Note the fibrofatty proliferation in the adjacent mesentery
With the exception of the cine and diffusion-weighted sequences, all scans are breath-hold and carried out in both the axial and coronal planes. The imaging parameters for our MRE protocol, and the order the sequences are performed in, are shown in Table 1.

\section{Rationale for MR sequences used in MRE}

The True FISP (fast imaging with steady state precession) sequence consists of a balanced gradient echo sequence where image contrast is dependent on $\mathrm{T} 2 * / \mathrm{T} 1$ ratio. It gives highcontrast, predominantly $\mathrm{T} 2 *$-weighted images. True FISP eliminates phase shifts caused by motion and thus both fluid and blood appear bright. It is a fast acquisition due to a short repetition time (TR) and echo time (TE) with each image acquired in a few hundred milliseconds. Susceptibility artefact occurs with the presence of intraluminal gas or ferromagnetic material, leading to image distortion. Off-resonance artefacts occur in the presence of a non-uniform magnetic field, resulting in banding artefact (alternating stripes) at the periphery of the image. Chemical-shift artefact results in a 'black boundary' effect around structures (Fig. 1). This makes mesenteric nodes and vessels more conspicuous but may impede assessment of bowel wall thickening. This sequence provides good delineation between the bowel and the mesentery.

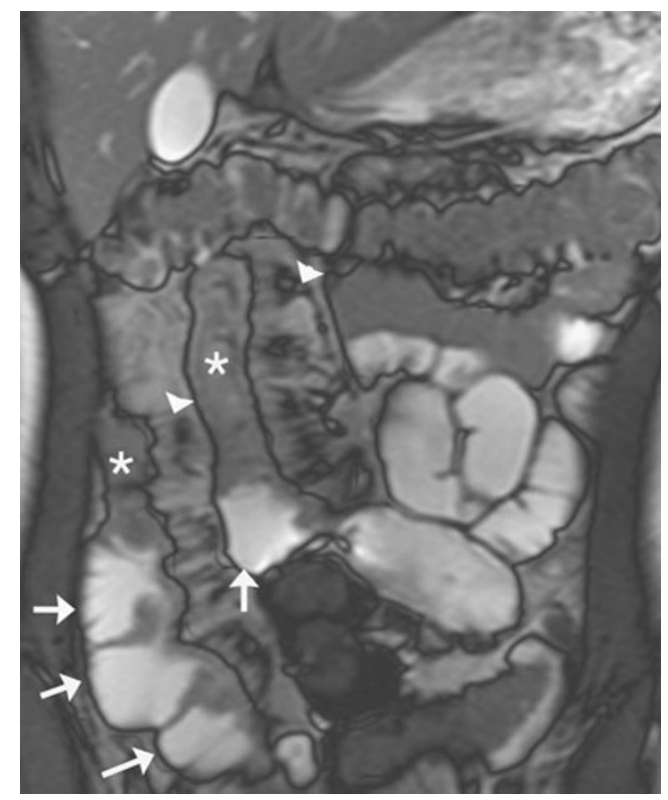

Fig. 5 Example of pseudosacculation: coronal True FISP image shows two long skip lesions in the mid to distal ileum (asterisk) demonstrating mural thickening, luminal narrowing and prominence of the vasa recta (arrowheads) with small nodes seen within the mesentery. There are multiple pseudosacculations (arrows) on the antimesenteric border due to asymmetric involvement of the bowel wall. Mesenteric fibrofatty proliferation separates the involved bowel loops. Appearances suggest a mixture of active and fibrostenotic disease 
Table 3 Comparison of active inflammation and fibrostenotic disease on MRE

\begin{tabular}{lll}
\hline Features on MRE & Active inflammation & Fibrostenotic disease \\
\hline Mural thickness & Moderate & Mild \\
Mural enhancement & Avid & Mild \\
Stratified mural enhancement & Yes & Variable \\
Mural oedema & Yes & Mild/absent \\
Increased mesenteric vascularity/adenopathy & Yes & No \\
Mesenteric phlegmon, abscess & Present in penetrating disease & No \\
Fistulae & Present in penetrating disease & Sometimes present \\
Fibrofatty proliferation & Sometimes present & Yes \\
\hline
\end{tabular}

The cine True FISP sequence involves repeatedly imaging the abdomen in the coronal plane sequentially from front to back over a period of 2 min. Twenty images are acquired in each coronal plane. Inflamed loops of bowel will demonstrate decreased peristalsis. An inflammatory small bowel stenosis should open up on cine imaging, compared with a fibrotic stricture where the stenosis will be constant and associated with more marked pre-stenotic dilatation.
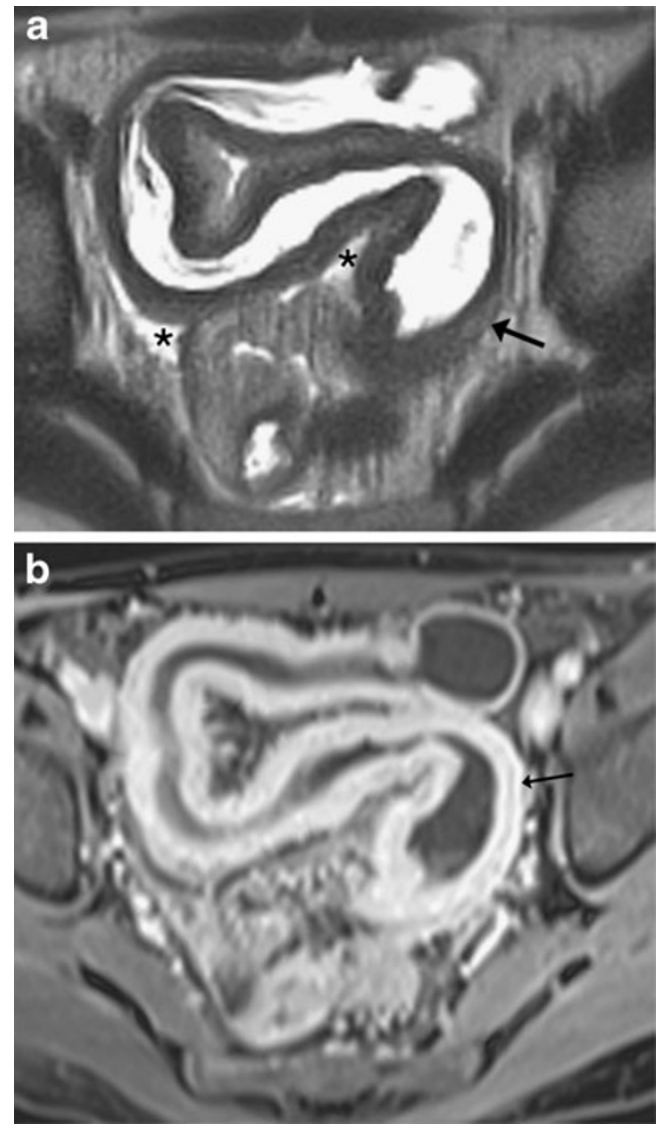

Fig. 6 Patient with active distal ileal Crohn's disease: a) axial HASTE image shows bowel wall is of intermediate signal (arrow) due to submucosal oedema. Mesenteric oedema (asterisk) is also noted; b) corresponding axial $\mathrm{T} 1$ fat-saturated post-contrast image shows avid stratified pattern of mural enhancement (arrow)
Diffusion-weighted imaging (DWI) has recently been added to our MRE protocol with parameters as shown in Table 1 . Three $b$ values $(b=0,100$ and 800$)$ are used with axial images through the upper and lower abdomen obtained. The purpose of this is to help identify actively
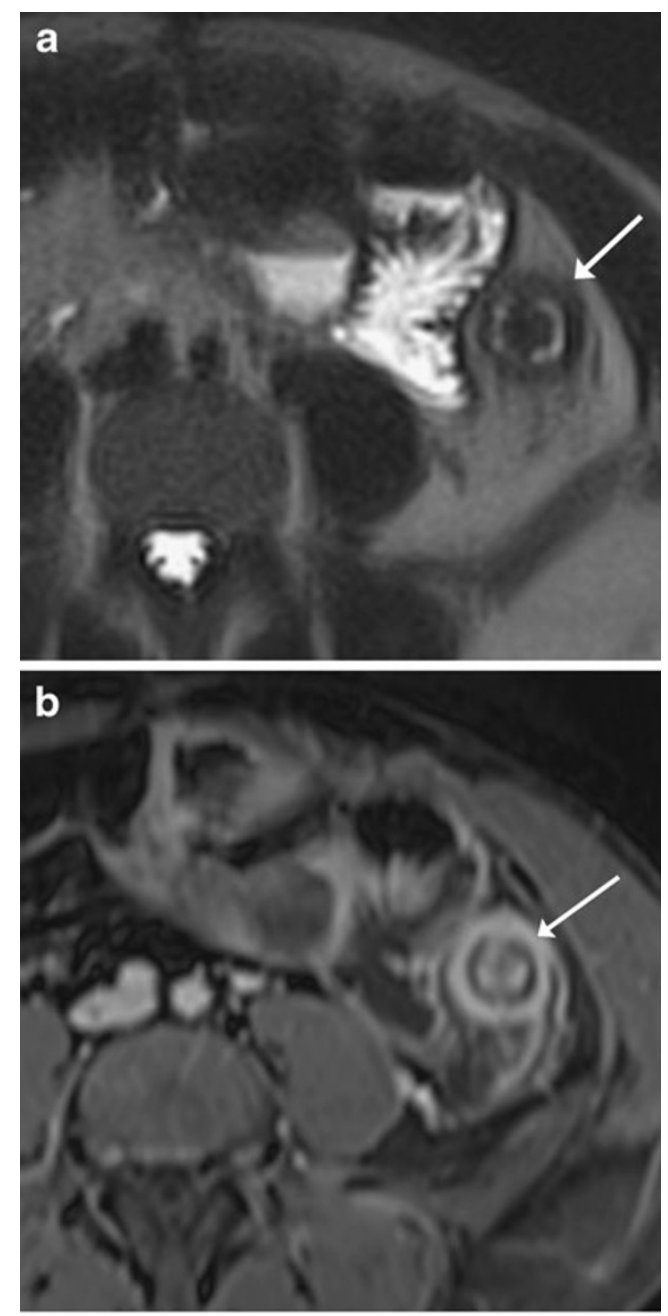

Fig. 7 Patient with active distal colitis: a axial HASTE image shows very high signal in the submucosa in keeping with oedema (arrow); b corresponding axial $\mathrm{T} 1$ fat-saturated post-contrast image shows avid stratified pattern of mural enhancement (arrow) 
inflamed loops of bowel by the presence of restricted diffusion within the affected bowel wall.

The post-contrast sequence involves a three-dimensional (3D) T1-weighted fat-saturated spoiled gradient echo sequence (Fig. 2) performed $70 \mathrm{~s}$ following hand injection of $0.2 \mathrm{ml} / \mathrm{kg}$ $(0.1 \mathrm{mmol} / \mathrm{kg}$ ) intravenous Gadoteric acid (Dotarem, Guerbet). Gadolinium is not given in patients with chronic renal impairment, due to the potential long-term risk of nephrogenic systemic fibrosis. A pre-contrast sequence is routinely performed. Some institutions use a dynamic post-contrast coronal acquisition; for example, a volume interpolated breath-hold examination (VIBE) can be performed in the arterial ( $30 \mathrm{~s}$ - optional) and portal venous (60-70 s) phases using bolus triggering once contrast reaches the descending aorta. With a 3D sequence, a radiofrequency pulse excites a thick volume of tissue rather than a thin 2D section, with increased spatial resolution. The $3 \mathrm{D}$ sequence is, however, sensitive to motion artefact. Bowel peristalsis is reduced by the prior intravenous administration of a spasmolytic. This usually consists of $20 \mathrm{mg}$ intravenous hyoscine-N-butylbromide (buscopan). Buscopan is routinely given unless contra-indicated (e.g. history of cardiac arrhythmia, narrow angle glaucoma or prostatism). In instances when this cannot be administered, $1 \mathrm{mg}$ intravenous glucagon is given as an alternative unless patients have a known hypersensitivity to glucagon or a history of phaeochromocytoma.. The purpose of this sequence is to assess bowel wall and mesenteric nodal enhancement and to evaluate for the presence of any rimenhancing fluid collections.

The HASTE (half-Fourier acquired single-shot fast spin echo) sequence is a fast sequence consisting of heavily T2-

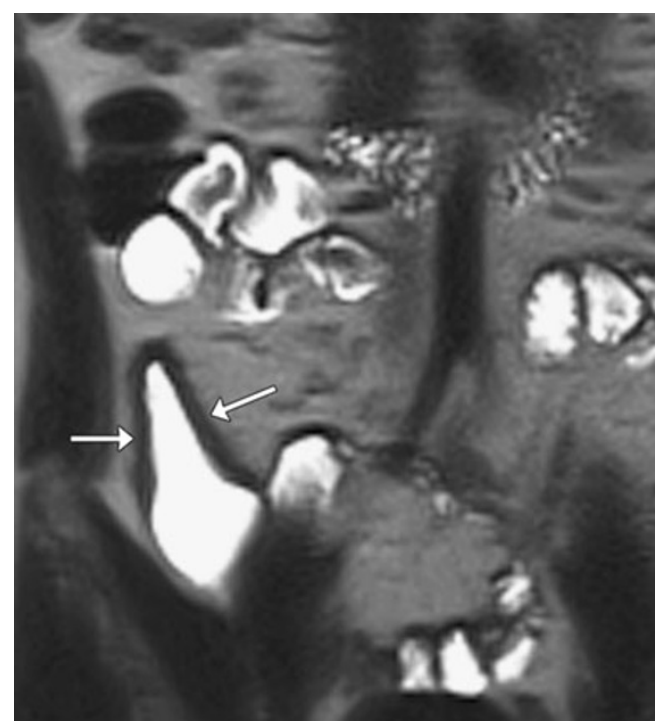

Fig. 8 Example of fibrostenotic disease: coronal HASTE image shows a stricture in the neoterminal ileum in a patient with previous ileocolic resection; this is of intermediate to low signal due to little mural oedema (arrows); there is pre-stenotic dilatation weighted images. It is a pulse sequence with a very long echo train, where each echo is individually phase encoded. The partial Fourier technique assumes symmetry of raw data in K-space, to reduce data acquisition time. However, this results in a reduced signal-to-noise ratio. It is good at depicting fluid, but other tissues with short $\mathrm{T} 2$ values are associated with blurring artefact. It is sensitive to intraluminal flow voids (Fig. 3) and is thus performed after the intravenous administration of a spasmolytic. The HASTE sequence is used to assess for mural oedema. The optional use of fat saturation on one of the T2-weighted sequences (either the True FISP or HASTE sequence) allows differentiation between submucosal fat and oedema which both appear bright on T2-weighted images. Fat saturation also increases the conspicuousness of oedematous bowel loops.
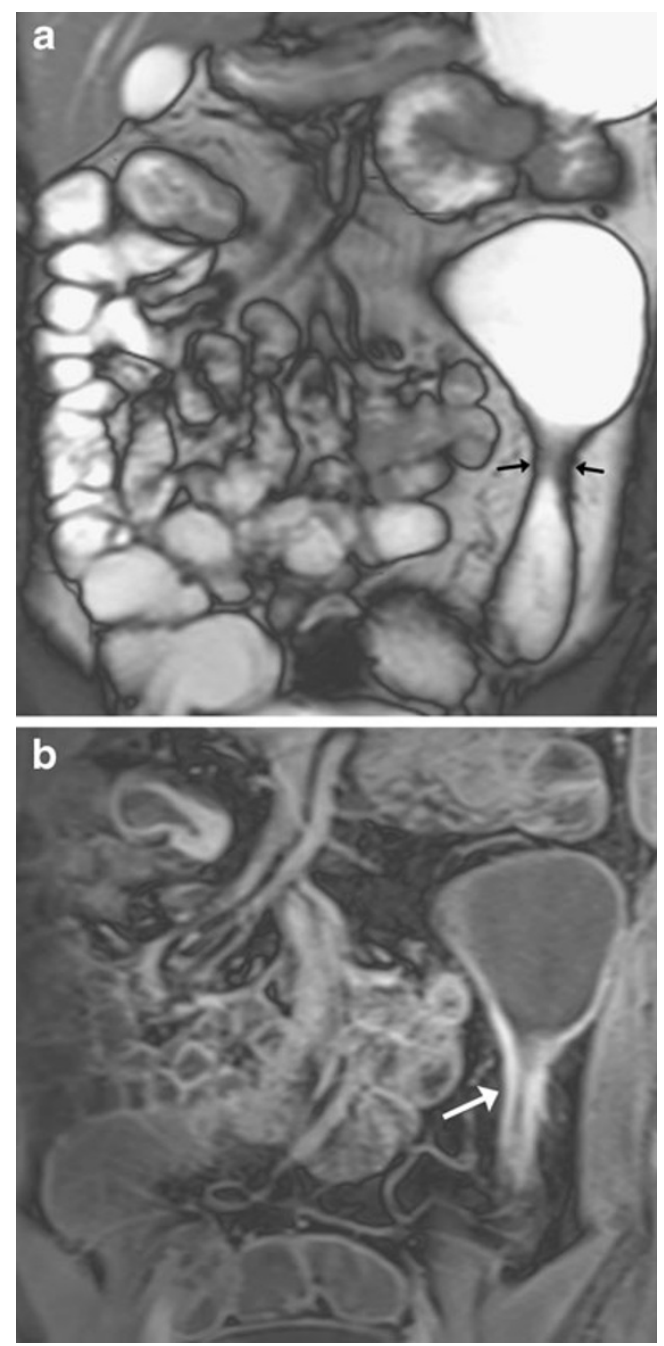

Fig. 9 Patient with fibrostenotic disease: a coronal True FISP image showing short fibrotic stricture (arrows) in the mid descending colon with marked pre-stenotic dilatation; b corresponding homogeneous mural enhancement is seen in this skip lesion (arrow) 


\section{Comparison with other imaging techniques}

Other radiology techniques used to image the small bowel include barium follow-through, CT enterography, ultrasound and nuclear medicine techniques (such as positron emission tomography [PET]-CT and white cell scintigraphy).

With regards to the use of PET or PET-CT in the evaluation of $\mathrm{CD}$, the sensitivity in the detection of active inflammation ranges between $73 \%$ and $90 \%$ when compared with clinical, endoscopic or biological markers of disease activity $[5,6]$. Using PET alone, there is poor disease localisation, and specificity can sometimes be low [7], as other pathological or physiological processes may lead to increased bowel fluorodeoxyglucose (FDG) uptake. The main disadvantage of PET-CT is the use of ionising radiation; this is clearly not ideal in young patients who may require repeated imaging. It is also more time consuming to perform than CT or MRI alone. Hence for these reasons, PET-CT is not routinely used in the assessment of patients with $\mathrm{CD}$.

A recent meta-analysis of 33 studies using ultrasound, MRE, scintigraphy and CT in the diagnosis of $\mathrm{CD}$, showed no significant difference in the mean sensitivity [8]. All these techniques showed a high sensitivity $(>84 \%)$ and specificity $(90 \%)$ on a per patient basis [8]. Hence the preference is based on availability and consideration of radiation exposure.

Enteroclysis is a technique whereby oral contrast is directly infused into the small bowel via a nasojejunal catheter at a high flow rate (e.g. $80 \mathrm{ml} / \mathrm{min}$ ) in order to obtain optimal small bowel distension. Depending on the oral contrast agent used and preference of the radiologist, the small bowel can then be imaged using either fluoroscopy, CT or MRI. MR enteroclysis gives better mucosal detail than MRE [9] and is thus more likely to detect subtle aphthous or other types of mucosal ulceration. However, in the assessment of ileal CD, MRE has shown an equivalent accuracy and reproducibility to MR enteroclysis [10].

In addition to ileocolonoscopy, the gastroenterologist can use other techniques to evaluate the small bowel. Double balloon enteroscopy can examine the whole of the small bowel, either under conscious sedation or general anaesthesia. Video (or wireless) capsule endoscopy (VCE) involves the ingestion of a capsule that contains a camera and transmitter, which is propelled along the bowel by peristalsis. A video recorder carried on the patient records the signals and a computer later processes the data. A recent meta-analysis [11] looking at nine studies comparing VCE to other techniques in the diagnosis of CD (i.e. ileocolonoscopy, CT enterography/enteroclysis, push enteroscopy and MRI) showed VCE gave a greater diagnostic yield due to its ability to detect mucosal abnormalities. However VCE is not possible in stricturing disease, hence radiological imaging is usually required first to exclude this (unless a patency capsule is used).
Table 2 shows the advantages and disadvantages of each technique when compared with MRE.

\section{Problem-solving in small bowel CD}

The multidisciplinary team meeting (consisting of the radiologist, gastroenterologist, histopathologist and surgeon) is an important forum where imaging, histopathology and clinical status of the patient can all be reviewed, in order to determine the most appropriate management. The following important questions can be addressed on MRE: (1) extent of small and large bowel involvement at first presentation; (2) distinction between active inflammatory and fibrotic stricturing disease; (3) recognition of penetrating disease with the development of extramural complications; (4) evaluation of response to medical therapy; (5) detection of recurrent disease following surgery. Each of these questions will be addressed in turn.

\section{Extent of bowel involvement at first presentation}

The hallmark of $\mathrm{CD}$ on cross-sectional imaging is bowel wall thickening (Fig. 4a, b) (between 4 to $12 \mathrm{~mm}$ ), usually in association with luminal stenosis. Normal bowel wall thickness, when adequately distended, should not exceed $3 \mathrm{~mm}$. Mural thickening can be appreciated on all sequences. The commonest site of involvement is the terminal ileum (sometimes with contiguous disease in the caecum). Discontinuous

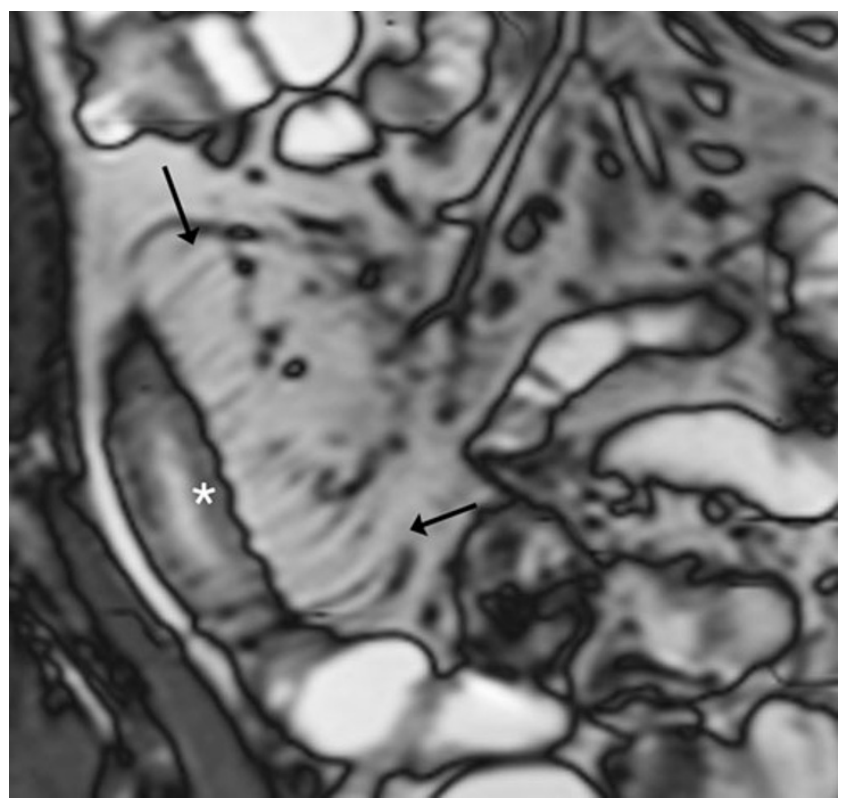

Fig. 10 Example of comb sign in active inflammation: coronal True FISP image shows multiple linear low signal structures extending to the bowel wall in keeping with engorged vasa recta (arrows) supplying the thickened terminal ileum (asterisk). Note the fibrofatty proliferation within the adjacent mesentery, separating the inflamed terminal ileum from adjacent loops of bowel, with small mesenteric nodes also present 
Fig. 11 Example of DWI in active inflammation: a axial T1 fatsaturated post-contrast image shows thickened enhancing ileum with typical stratified enhancement pattern (arrow); b corresponding DWI $(b=800)$ and $\mathbf{c}$ ADC map confirm restricted diffusion with persistent high signal (arrow) on the DWI and low signal (arrow) on the ADC map $(\mathrm{ROI}=1,200)$

skip lesions may be seen more proximally in the small bowel or within the colon. If there is suboptimal distension (as is sometimes the case, especially with jejunal loops), disease may be overestimated or underestimated. Early disease, characterised by mucosal changes only, may also not be appreciated on MRE. The evaluation of all sequences may help clarify the extent of bowel involvement, as fluid distension in the small bowel will vary over time. Strictures can also be distinguished from peristalsis on the cine sequence. Involvement of the bowel wall may be symmetrical or asymmetrical, where greater involvement of the mesenteric border leads to pseudo-sacculation (Fig. 5). With repeated episodes of acute inflammation, mesenteric fibrofatty proliferation ('creeping fat'/'fat wrapping') tends to develop along the mesenteric border of the involved segment of bowel (Fig. 5); this is a helpful additional feature on MRE denoting the site of disease.

Distinction between active inflammatory and stricturing disease

A key question in the management of patients with $C D$ is the assessment of disease activity. Active inflammation (characterised clinically by acute clinical symptoms and raised inflammatory markers) is usually treated medically (unless there are extra-mural complications), whilst fibrostenotic disease (characterised by obstructive symptoms) often requires surgery. In addition to dietary approaches, there are a number of drugs that can be used to induce and/or maintain remission in $\mathrm{CD}$ [12]. In the last decade, anti-tumour necrosis factor alpha (TNF)- $\alpha$ antibodies (infliximab, adalimumab) have become an established and highly effective treatment for moderate to severe CD.

Endoscopy (with the use of an endoscopic severity score) with or without biopsy can be used to directly assess the degree of inflammation in the bowel. However, this is an invasive technique, which is restricted to the colon and distal $20-30 \mathrm{~cm}$ of ileum. There are several surrogate markers for disease activity that clinicians have traditionally used (including the Crohn's Disease Activity Index [CDAI], C-reactive protein [CRP] and more recently faecal markers such as faecal calprotectin). All of these markers, however, have limitations. In a meta-analysis of seven studies (140 patients) looking at MRI to determine disease activity, MRI correctly graded disease activity in $91 \%$ of cases with frank disease [13]. Recently, attempts have been made to derive an MR-based activity score (using MR features such as mural
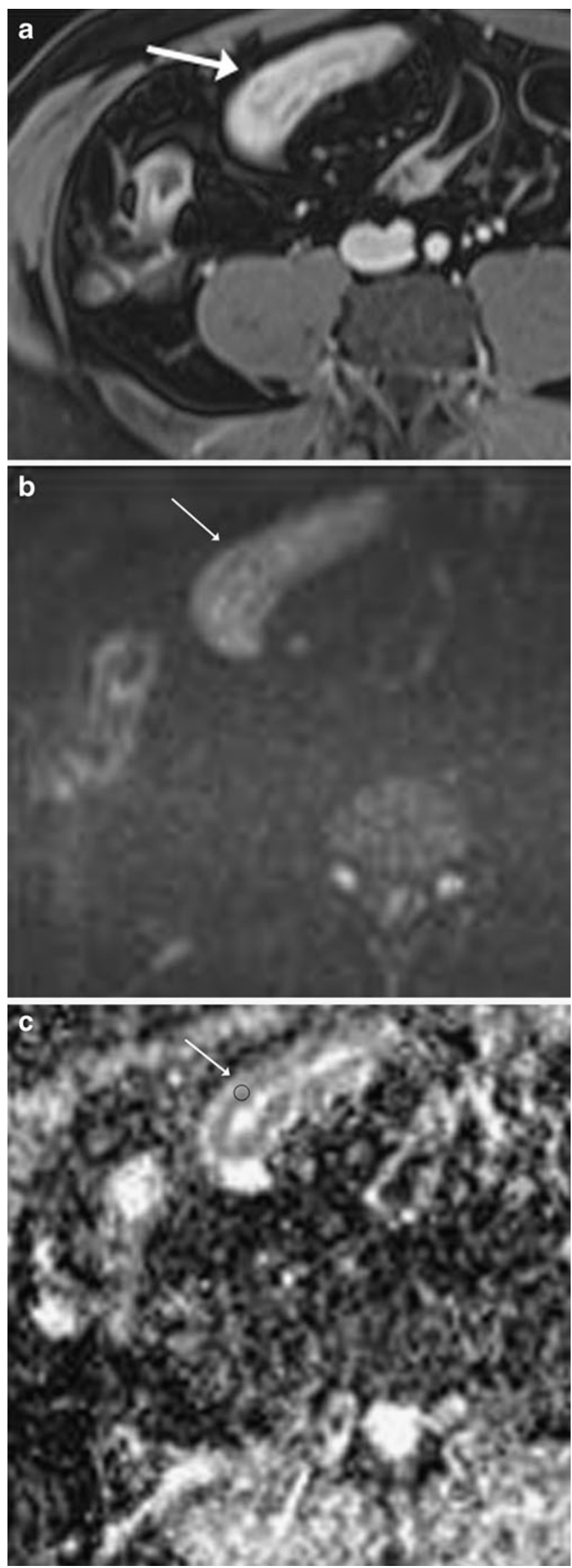
thickness and oedema) to assess disease activity, validated with ileocolonoscopy and histology as the standard of reference $[14,15]$.

When using MRE to assess activity of disease, the following features can be evaluated. These have been summarised in Table 3 .

\section{Degree of mural thickening}

It has been shown [16] that active inflammation (as defined by findings of inflamed mucosa or inflammatory infiltrates on endoscopy or histopathology respectively) has a greater degree of mural thickening on MRE (Fig. 4a, b) compared with chronic inflammation or fibrostenotic disease (mean maximal wall thickness $6.7 \mathrm{~mm}$ versus $3.3 \mathrm{~mm}$ respectively). A direct positive correlation has been demonstrated between the degree of acute inflammation on histopathology (defined by an acute inflammatory score) and the degree of mural thickening on MRE [17]. This is not surprising as mural thickening is probably due to a combination of oedema and inflammatory infiltrates.

\section{Bowel wall signal intensity on T2-weighted images}

The affected bowel wall in active inflammation demonstrates higher signal intensity on T2-weighted images due to the presence of mucosal or submucosal oedema (Figs. 6a, 7a). Good correlation has been shown between mural hyperintensity on T2-weighted images, biological parameters (such as CDAI and CRP) and histopathology [17, 18]. In fibrostenotic disease, the bowel wall appears of lower signal due to the presence of fibrosis (Figs. 8, 9a). Sometimes in chronic disease, submucosal fat deposition occurs, which is also of high signal on T2-weighted images. As mentioned earlier, the use of a fat-suppressed T2-weighted sequence can thus be helpful in distinguishing submucosal fat from oedema.

\section{Degree of mural enhancement}

Active inflammation results in an increase in bowel wall enhancement compared with adjacent uninvolved bowel [19-23]. Less avid mural enhancement is seen in fibrostenotic disease. Some studies have used dynamic contrast enhanced MRI [20-23] to obtain either semi-quantitative
Fig. 12 Complications of penetrating disease: a coronal True FISP image showing an enteroenteric fistula (arrows) between an inflamed segment of mid ileum (white asterisk) and non-inflamed terminal ileum (black asterisk); b Axial True FISP image in a different patient showing an enterocutaneous fistula (arrows) between a loop of inflamed thickened small bowel (asterisk) and skin; c axial True FISP image in a different patient showing the 'star' sign between adjacent loops of bowel, highly suggestive of enteroenteric fistulae (arrows); $\mathbf{d}$ coronal T1 fat saturated post contrast image in a different patient again showing multiple converging enhancing loops of small bowel suggestive of enteroenteric fistulae (arrows)
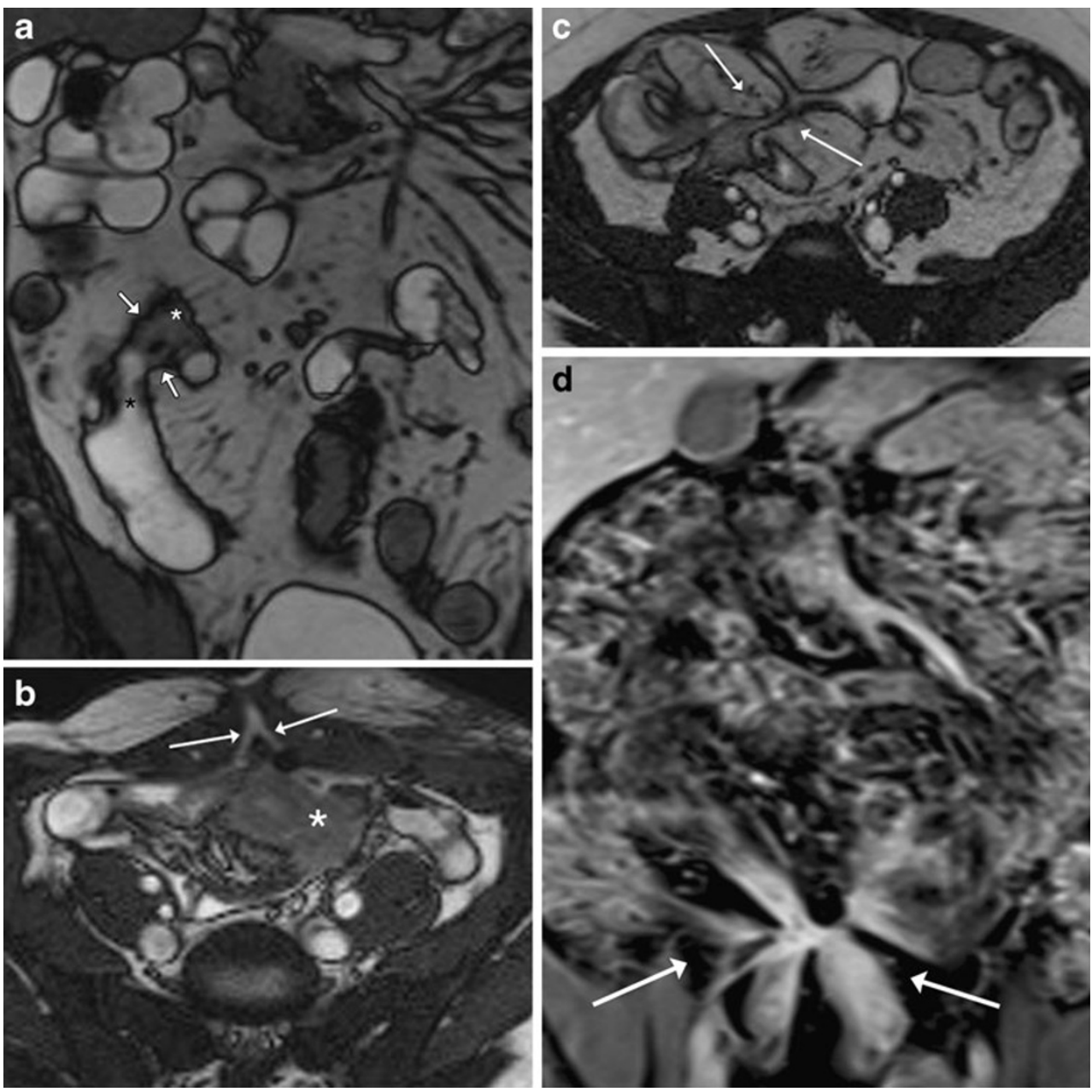


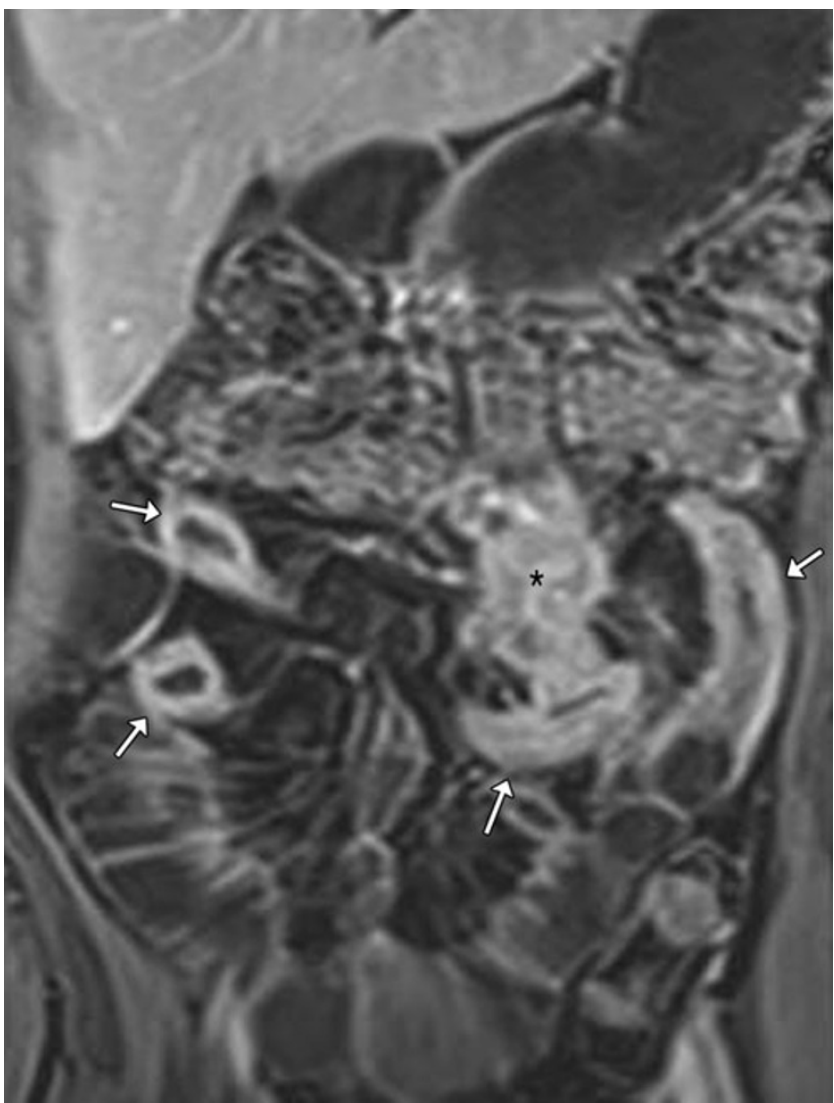

Fig. 13 Complications of penetrating disease: coronal T1 fat-saturated post-contrast image showing multiple enhancing skip lesions (arrows) and enhancing mesenteric phlegmon (asterisk)

or quantitative measurements of enhancement. Results suggest that inflamed bowel segments have steeper initial enhancement

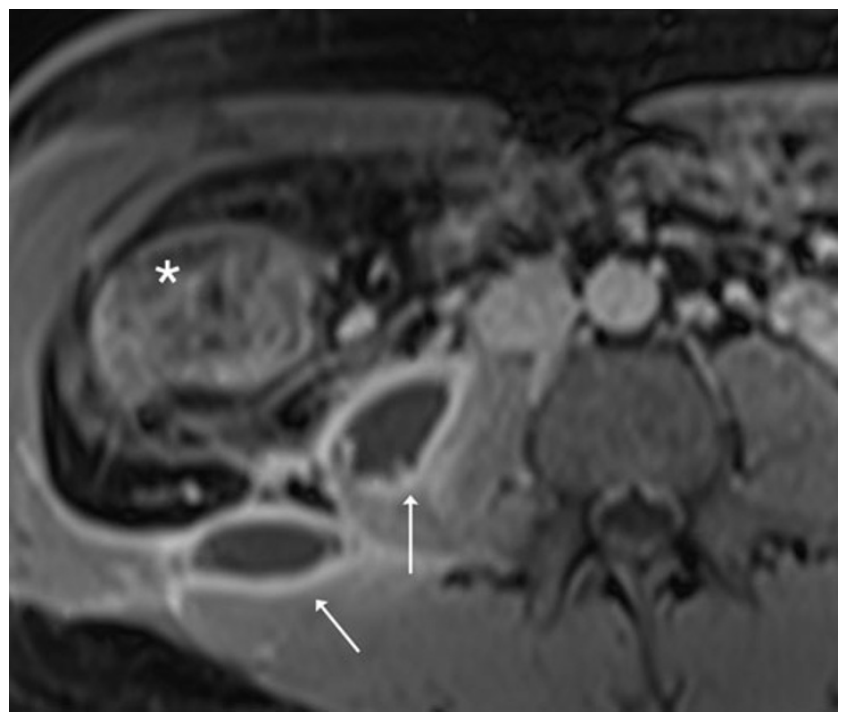

Fig. 14 Complication of penetrating disease: patient with active colitis showing circumferential mural thickening and stratified mural enhancement of the proximal colon (asterisk), complicated by an ileopsoas abscess (arrows) slopes, and faster volume transfer coefficients (Ktrans) [21, 23]. A linear relationship between the degree of bowel wall enhancement and degree of inflammatory activity on histopathology has, however, not been clearly established [17].

\section{Pattern of mural enhancement}

Active inflammation may show either increased mucosal enhancement or a stratified pattern (Fig. 6b, 7b) of mural enhancement $[17,18]$. The latter is due to the presence of submucosal oedema, where there is avid enhancement of the mucosa and the muscularis propria/serosa but relatively reduced enhancement of the submucosa. Chronic inflammation and fibrostenotic disease may show either a heterogeneous, homogeneous (Fig. 9b) [24] or stratified pattern of enhancement [18]. It is postulated [17] that the stratified pattern of enhancement seen in fibrostenotic disease may be due to the presence of active disease on the background of mural fibrotic change or the presence of mural fibrosis itself (which theoretically would then show delayed enhancement).

\section{Pre-stenotic dilatation}

Dilatation can occur upstream to either an inflammatory stenosis or fibrostenotic stricture (Fig. 9a, b). This is significant when the small bowel diameter is $>3 \mathrm{~cm}$. As mentioned earlier, cine imaging is helpful in distinguishing between active inflammatory and fibrotic strictures.

\section{'Comb'sign and mesenteric adenopathy}

In active inflammation, the vascular arcades (vasa recta) supplying the involved bowel segment become engorged.

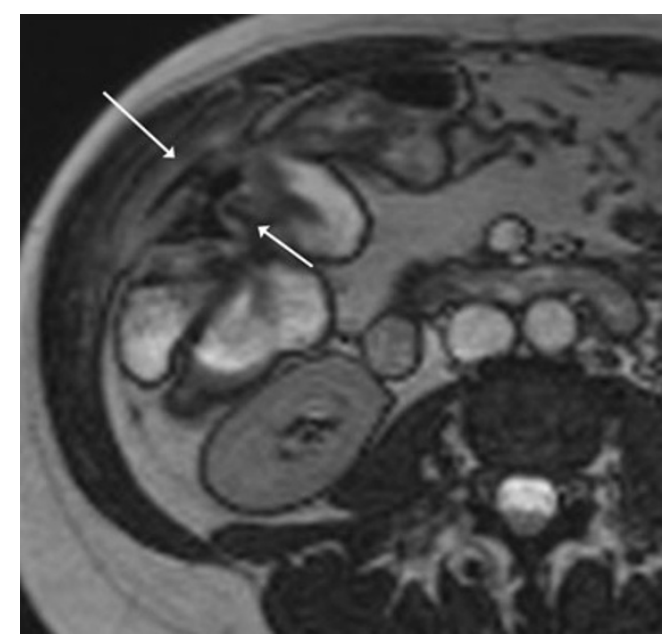

Fig. 15 Complication of penetrating disease: patient with local perforation due to penetrating Crohn's disease of the proximal colon-a thick walled air-containing cavity is seen extending into the anterior abdominal wall (arrows) 
These are straight vessels that extend perpendicular to the bowel wall within the mesentery and resemble the teeth of a comb. They appear as low signal linear structures on True FISP images (Fig. 10) with enhancement post-contrast. Prominent reactive enhancing mesenteric nodes (up to $8 \mathrm{~mm}$ in short axis diameter) will also be seen in active inflammation (Figs. 5, 10). If larger volume adenopathy is seen, the development of lymphoma needs to be excluded.

\section{Restricted diffusion}

In the last 5 years, there have been several studies [25-27] that have suggested that DWI may have an adjunctive role in the assessment of disease activity in affected bowel. Either visual assessment of DWI or quantitative assessment of the ADC value can be made. Restricted diffusion in affected bowel segments is more likely in active inflammation (Fig. 11a-c) compared to fibrostenotic disease [25-27].

Recognition of penetrating disease and development of extramural complications

\section{Sinus and fistula formation}

Blind ending sinus tracts and fistulae may develop in active CD due to transmural bowel inflammation and penetrating ulceration. Enteroenteric (Fig. 12a), enterocolic, enterovesical and enterocutaneous (Fig. 12b) fistulae may arise. These are usually well demonstrated on the True FISP and HASTE sequences due to high signal fluid content within the tracts, but will also be seen as rim-enhancing low-signal tracts on the post-contrast images. The 'star' sign (Fig. 12c, d) is where multiple converging loops of inflamed bowel are seen and is suggestive of enteroenteric fistulae. It has been shown that MRE has an overall sensitivity for sinus tracts and fistulae of $>75 \%$ and specificity of $100 \%$, when compared with MR enteroclysis and conventional enteroclysis as the reference standards [6].

\section{Mesenteric phlegmon and abscess collections}

Active inflammation can result in the formation of small enhancing phlegmons within the mesentery (Fig. 13). Rim enhancing heterogeneous abscess collections (Fig. 14) can also develop within the abdomen, possibly secondary to localised bowel wall perforation (Fig. 15). These abscesses are important to identify, as antibiotics and/or percutaneous drainage may be required.

\section{Evaluation of response to medical therapy}

MRE is a useful tool in the assessment of treatment response following medical therapy. It has been shown that when MRI is performed during an acute relapse and then in remission, a reduction in both mural contrast enhancement and in mural thickness in affected segments is seen [28]. However, luminal stenosis may persist. As stated earlier, biologics such as infliximab and adalimumab have been used to treat patients with $\mathrm{CD}$ resistant to other therapies with mucosal healing as a key treatment goal. From our experience, MRE can be used to show a significant reduction in inflammatory activity (as demonstrated by reduced mural thickening, oedema and enhancement) following treatment with such agents (Fig. 16a,
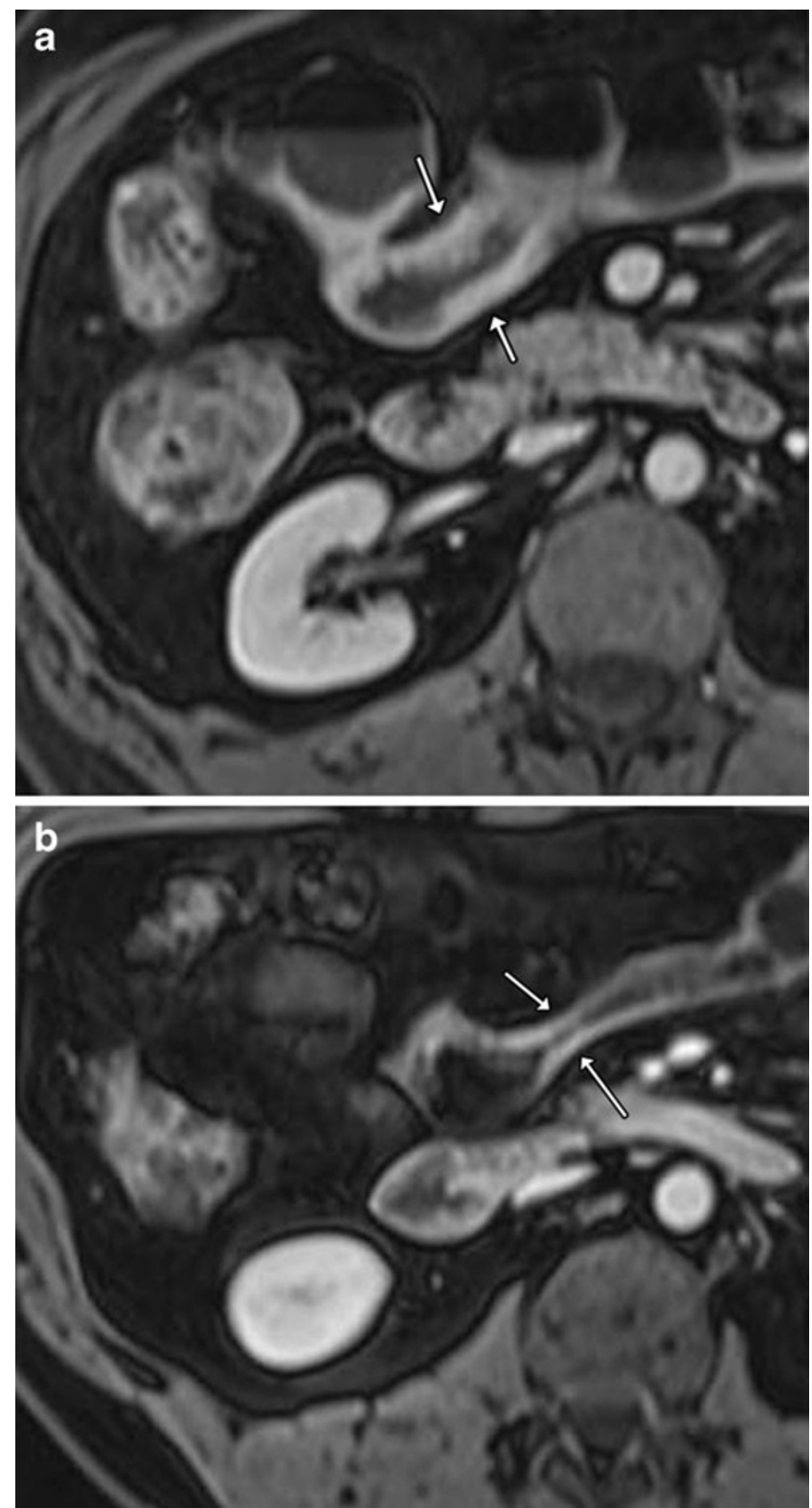

Fig. 16 Typical example of response to treatment with a biologic: a axial T1 fat-saturated post-contrast image shows a thickened avidly enhancing segment of midileum with stratified pattern of enhancement (arrows) in keeping with active inflammation; $\mathbf{b}$ axial T1 fat-saturated post-contrast image following 8 months of infliximab shows inflammation has been downstaged with reduction in mural thickening and enhancement which is now homogeneous (arrows) 
b). DWI may have a role in response assessment with a predicted decrease in the degree of restricted diffusion. However, as yet there are no published papers on this subject.

\section{Detection of recurrent disease following surgery}

As the terminal ileum is the commonest site of disease in CD, the majority of patients eventually requiring surgery typically have an ileocolic resection. Stricturoplasties may also be performed in short $(<3 \mathrm{~cm})$ fibrotic strictures causing obstructive symptoms. Patients are routinely re-evaluated by ileocolonoscopy at 6-12 months post-resection. Post-surgical recurrence, defined as the appearance of new lesions on endoscopy, radiology or pathology, occurs in $80 \%$ of patients at the site of the neoterminal ileum/anastomosis (Fig. 8) within the 1st year following resection [29, 30]. Rutgeerts et al. [29] have shown that patients with absent or very mild lesions on endoscopy following surgery have a very good prognosis, whereas patients with more severe lesions on endoscopy develop early clinical recurrence. Limitations of ileocolonoscopy are that intubation of the neoterminal ileum may not be possible due to stenosis, more proximal small bowel disease will not be assessed and extramural complications will not be demonstrated. Recently both MR enteroclysis [31] and MR enterography [32] have been suggested as useful alternatives, showing good concordance with ileocolonoscopy in the detection of recurrence and in the differentiation of high and low risk patients.

\section{Conclusion}

Imaging has an important role in the management of CD. Although various imaging modalities are available, MRE is increasingly becoming the first line investigation for such patients. It can be useful at both initial diagnosis and followup. It is the preferred investigation due to its lack of ionising radiation and its ability to depict both mural and extramural complications. MRE can be performed prior to VCE in excluding strictures where VCE can then be used to detect subtle mucosal disease. In combination with blood and faecal biomarkers and endoscopy, MRE can assist the treating clinician in distinguishing between inflammatory stenoses amenable to medical therapy and fibrostenotic disease requiring surgery. The value of DWI remains to be defined, but it may well have an adjunctive role in the assessment of disease activity and response evaluation.

\section{References}

1. Loftus EV Jr (2004) Clinical epidemiology of inflammatory bowel disease: incidence, prevalence, and environmental influences. Gastroenterology 126:1504-1517
2. Van Assche G, Dignass A, Panes J, Beaugerie L, Karagiannis J, Allez M, Ochsenkühn T, Orchard T, Rogler G, Louis E, Kupcinskas L, Mantzaris G, Travis S, Stange E, European Crohn's and Colitis Organisation (ECCO) (2010) The second European evidence-based Consensus on the diagnosis and management of Crohn's disease: Definitions and diagnosis. J Crohns Colitis 4:7-27

3. Hafeez R, Punwani S, Boulos P, Bloom S, McCartney S, Halligan S, Taylor SA (2011) Diagnostic and therapeutic impact of MR enterography in Crohn's disease. Clin Radiol 66:1148-1158

4. Cronin CG, Lohan DG, Mhuircheartaigh JN, McKenna D, Alhajeri N, Roche C, Murphy JM (2008) MRI small-bowel follow-through: prone versus supine patient positioning for best small-bowel distention and lesion detection. AJR Am J Roentgenol 191:502-506

5. Lemberg DA, Issenman RM, Cawdron R, Green T, Mernagh J, Skehan SJ et al (2005) Positron emission tomography in the investigation of pediatric inflammatory bowel disease. Inflamm Bowel Dis 11:733-738

6. Louis E, Ancion G, Colard A, Spote V, Belaiche J, Hustinx R (2007) Noninvasive assessment of Crohn's disease intestinal lesions with (18)F-FDG PET/CT. J Nucl Med 48:1053-1059

7. Löffler M, Weckesser M, Franzius C, Schober O, Zimmer KP (2006) High diagnostic value of 18 F-FDG-PET in pediatric patients with chronic inflammatory bowel disease. Ann N Y Acad Sci 1072:379-385

8. Horsthuis K, Bipat S, Bennink RJ, Stoker J (2008) Inflammatory bowel disease diagnosed with US, MR, scintigraphy, and CT: meta-analysis of prospective studies. Radiology 247:64-79

9. Masselli G, Casciani E, Polettini E, Gualdi G (2008) Comparison of MR enteroclysis with MR enterography and conventional enteroclysis in patients with Crohn's disease. Eur Radiol 18:438-447

10. Negaard A, Paulsen V, Sandvik L, Berstad AE, Borthne A, Try K, Lygren I, Storaas T, Klow NE (2007) A prospective randomized comparison between two MRI studies of the small bowel in Crohn's disease, the oral contrast method and MR enteroclysis. Eur Radiol 17:2294-2301

11. Triester SL, Leighton JA, Leontiadis GI, Gurudu SR, Fleischer DE, Hara AK, Heigh RI, Shiff AD, Sharma VK (2006) A metaanalysis of the yield of capsule endoscopy compared to other diagnostic modalities in patients with non-stricturing small bowel Crohn's disease. Am J Gastroenterol 101:954-964

12. Triantafillidis JK, Merikas E, Georgopoulos F (2011) Current and emerging drugs for the treatment of inflammatory bowel disease. Drug Des Devel Ther 6:185-210

13. Horsthuis K, Bipat S, Stokkers PC, Stoker J (2009) Magnetic resonance imaging for evaluation of disease activity in Crohn's disease: a systematic review. Eur Radiol 19:1450-1460

14. Rimola J, Ordás I, Rodriguez S, García-Bosch O, Aceituno M, Llach J, Ayuso C, Ricart E, Panés J (2011) Magnetic resonance imaging for evaluation of Crohn's disease: validation of parameters of severity and quantitative index of activity. Inflamm Bowel Dis 17:1759-1768

15. Steward MJ, Punwani S, Proctor I, Adjei-Gyamfi Y, Chatterjee F, Bloom S, Novelli M, Halligan S, Rodriguez-Justo M, Taylor SA (2011) Non-perforating small bowel Crohn's disease assessed by MRI enterography: derivation and histopathological validation of an MR-based activity index. Eur J Radiol Sep 14. [Epub ahead of print]

16. Koh DM, Miao Y, Chinn RJ, Amin Z, Zeegen R, Westaby D, Healy JC (2001) MR imaging evaluation of the activity of Crohn's disease. AJR Am J Roentgenol 177:1325-1332

17. Punwani S, Rodriguez-Justo M, Bainbridge A, Greenhalgh R, De Vita E, Bloom S, Cohen R, Windsor A, Obichere A, Hansmann A, Novelli M, Halligan S, Taylor SA (2009) Mural inflammation in Crohn disease: location-matched histologic validation of MR imaging features. Radiology 252:712-720

18. Maccioni F, Viscido A, Broglia L, Marrollo M, Masciangelo R, Caprilli R, Rossi P (2000) Evaluation of Crohn disease activity with magnetic resonance imaging. Abdom Imaging 25:219-228 
19. Miao YM, Koh DM, Amin Z, Healy JC, Chinn RJ, Zeegen R, Westaby D (2002) Ultrasound and magnetic resonance imaging assessment of active bowel segments in Crohn's disease. Clin Radiol 57:913-918

20. Florie J, Wasser MN, Arts-Cieslik K, Akkerman EM, Siersema PD, Stoker J (2006) Dynamic contrast-enhanced MRI of the bowel wall for assessment of disease activity in Crohn's disease. AJR Am J Roentgenol 186:1384-1392

21. Pupillo VA, Di Cesare E, Frieri G, Limbucci N, Tanga M, Masciocchi C (2007) Assessment of inflammatory activity in Crohn's disease by means of dynamic contrast-enhanced MRI. Radiol Med 112:798809

22. Röttgen R, Grandke T, Grieser C, Lehmkuhl L, Hamm B, Lüdemann L (2010) Measurement of MRI enhancement kinetics for evaluation of inflammatory activity in Crohn's disease. Clin Imaging 34:29-35

23. Oto A, Kayhan A, Williams JT, Fan X, Yun L, Arkani S, Rubin DT (2011) Active Crohn's disease in the small bowel: evaluation by diffusion weighted imaging and quantitative dynamic contrast enhanced MR imaging. J Magn Reson Imaging 33:615-624

24. Del Vescovo R, Sansoni I, Caviglia R, Ribolsi M, Perrone G, Leoncini E, Grasso RF, Cicala M, Zobel BB (2008) Dynamic contrast enhanced magnetic resonance imaging of the terminal ileum:differentiation of activity of Crohn's disease. Abdom Imaging 33:417-424

25. Oussalah A, Laurent V, Bruot O, Bressenot A, Bigard MA, Régent D, Peyrin-Biroulet L (2010) Diffusion-weighted magnetic resonance without bowel preparation for detecting colonic inflammation in inflammatory bowel disease. Gut 59:1056-1065

26. Kiryu S, Dodanuki K, Takao H, Watanabe M, Inoue Y, Takazoe M, Sahara R, Unuma K, Ohtomo K (2009) Free-breathing diffusionweighted imaging for the assessment of inflammatory activity in Crohn's disease. J Magn Reson Imaging 29:880-886

27. Oto A, Zhu F, Kulkarni K, Karczmar GS, Turner JR, Rubin D (2009) Evaluation of diffusion-weighted MR imaging for detection of bowel inflammation in patients with Crohn's disease. Acad Radiol 16:597-603

28. Sempere GA, Martinez Sanjuan V, Medina Chulia E, Benages A, Tome Toyosato A, Canelles P, Bulto A, Quiles F, Puchades I, Cuquerella J, Celma J, Orti E (2005) MRI evaluation of inflammatory activity in Crohn's disease. AJR Am J Roentgenol 184:1829-1835

29. Rutgeerts P, Geboes K, Vantrappen G, Beyls J, Kerremans R, Hiele M (1990) Predictability of the postoperative course of Crohn's disease. Gastroenterology 99:956-963

30. Lee EC, Papaioannou N (1980) Recurrence following surgery for Crohn's disease. Clin Gastroenterol 9:419-438

31. Koilakou S, Sailer J, Peloschek P et al (2010) Endoscopy and MR enteroclysis: Equivalent tools in predicting clinical recurrence in patients with Crohn's disease after ileocolic resection. Inflamm Bowel Dis 16:198-203

32. Gallego Ojea JC, Echarri Piudo AI, Porta Vila A (2011) Crohn's disease: the usefulness of MR enterography in the detection of recurrence after surgery. Radiologia Mar 28 (Epub ahead of print) 